\title{
Low Preoperative Lymphocyte to Monocyte Ratio Serves as a Worse Prognostic Marker in Patients with Esophageal Squamous Cell Carcinoma Undergoing Curative Tumor Resection
}

\author{
Qian Song ${ }^{1}$, Jun-zhou $\mathrm{Wu}^{2}$, Sheng Wang ${ }^{3 凶}$ \\ 1. Department of Clinical Laboratory, Zhejiang Cancer Hospital, Key Laboratory of Head \& Neck Cancer Translational Research of Zhejiang \\ Province, Hangzhou, Zhejiang, People's Republic of China \\ 2. Cancer Research Institute, Zhejiang Cancer Hospital \& Key Laboratory Diagnosis and Treatment Technology on Thoracic Oncology of Zhejiang \\ Province, Hangzhou, Zhejiang, People's Republic of China \\ 3. Department of Clinical Laboratory, Zhejiang Cancer Hospital, Hangzhou, Zhejiang, People's Republic of China \\ $\square$ Corresponding author: Sheng Wang, Department of Clinical Laboratory, Zhejiang Cancer Hospital, Hangzhou, Zhejiang, 310022, People's Republic of China. \\ Tel: 86-571-88122229; Fax: 86-571-88122229; E-mail: wangsheng@zjcc.org.cn. \\ (C) Ivyspring International Publisher. This is an open access article distributed under the terms of the Creative Commons Attribution (CC BY-NC) license \\ (https://creativecommons.org/licenses/by-nc/4.0/). See http://ivyspring.com/terms for full terms and conditions.
}

Received: 2018.08.21; Accepted: 2019.04.03; Published: 2019.05.12

\begin{abstract}
Preoperative lymphocyte to monocyte ratio (LMR) has been considered a prognostic factor in various cancers. However, the application of LMR in the assessment of patients with esophageal squamous cell carcinoma (ESCC) remains controversial. This study aimed to investigate whether preoperative LMR could serve as a prognostic marker in patients with ESCC undergoing curative tumor resection. Medical records of 680 patients of ESCC after curative surgery without preoperative adjuvant therapy were obtained. The median of LMR was determined as the optimal cut off value. The association of LMR with clinical features of ESCC was analyzed using chi-square tests. Spearman's correlation coefficient was used to calculate the correlation. Disease-free survival (DFS) and overall survival (OS) stratified by LMR were evaluated using Kaplan-Meier method and log-rank test. The LMR was negatively correlated with sex $(r=-0.245, P<0.001)$. Low LMR $(L M R<3.17)$ predicted a shorter DFS and OS in patients with ESCC. Multivariate analyses revealed that LMR was independently correlated with DFS (hazard ratios $0.854 ; 95 \%$ confidence interval 0.768-0.949; $\mathrm{P}=0.003$ ) and OS (hazard ratios $0.864 ; 95 \%$ confidence interval 0.779-0.958; $P=0.006$ ). Our study indicated that low LMR could serve as an independent worse prognostic marker in patients with ESCC.
\end{abstract}

Key words: lymphocyte to monocyte, esophageal squamous cell carcinoma, prognosis

\section{Introduction}

Esophageal cancer is identified as the sixth leading cause of cancer-related mortality in the world [1]. In China, esophageal cancer is one of the most frequent malignant carcinomas and the fourth most common cause of cancer-related death [2]. Meanwhile, 90 percent of cases are esophageal squamous cell carcinomas (ESCC) [3]. Although significant advances achieved in multimodality therapies including surgery, chemotherapy and radiotherapy, patients with ESCC still face with unfavorable prognosis that the 5-year survival rate is only around 30\% [4-6]. Several factors are associated with the prognosis of ESCC, including clinicopathological characteristics such as TNM stage and tumor differentiation. Nevertheless, patients with the same TNM stage have disparate prognosis [7]. Therefore, it is imperative to identify new and accurate prognosis biomarkers of ESCC.

A growing number of studies have showed that cancer-related inflammation results in worse prognosis. Moreover, systemic inflammation plays an outstanding role in carcinoma development, 
progression and metastasis [8]. A variety of inflammatory biomarkers including neutrophil to lymphocyte ratio (NLR), C-reactive protein to albumin ratio (CAR) have been extensively studied in various cancers [9-11]. The lymphocyte to monocyte ratio (LMR) has been associated with worse prognosis in various cancers such as hepatocellular carcinoma, ovarian cancer and breast cancer [12-14]. However, because of the inconsistent results, whether LMR are associated with the prognosis in ESCC remains controversial [15-18]. Therefore, this retrospective study aimed to investigate whether preoperative LMR plays a key role in survival of patients with ESCC.

\section{Materials and Methods}

\section{Patient selection}

A total of 731 patients newly diagnosed with ESCC from Feb 2008 to Jan 2015 at the Zhejiang Cancer Hospital were selected for the research. The histological grades were determined based on the World Health Organization classification criteria. The inclusion standard as follows: (1) ESCC was pathologically confirmed; (2) received esophagectomy after diagnosis without preoperative chemotherapy or radiotherapy; (3) sufficient clinical information and follow-up data. 51 patients were excluded: 43 patients with preoperative chemotherapy, 7 patients with preoperative radiotherapy and 1 patient without preoperative LMR available. As result, 680 patients with ESCC were enrolled in the study.

The preoperative peripheral blood cell count was examined within 1 week prior to surgery. The LMR was calculated by dividing the absolute lymphocyte count with the absolute monocyte count. The conduct of this study was approved by the Ethics Committee of Zhejiang Cancer Hospital. All individual participants completed written informed consent.

\section{Blood-routine markers}

Patients were requested to be fasted after midnight prior to draw blood sample. On the morning, venous blood sample $(2 \mathrm{~mL})$ was drawn into the EDTA-K2 anticoagulative tubes. The lymphocyte and monocyte were examined by the SYSMEX XE-2100 (Sysmex, Kobe, Japan) Automatic Blood Cell Analyzer.

\section{Statistical analysis}

Categorical data are expressed as percentage and continuous data without a normal distribution as median and interquartile range. The lymphocyte, monocyte and LMR were analyzed as continuous variables and all clinicopathologic characteristics were counted as categorical variables.
The association of LMR with clinicopathological features of ESCC was analyzed using chi-square tests. The median of LMR was used to determine the cutoff value. Overall survival (OS) and disease-free survival (DFS) were calculated by the Kaplan-Meier method. The log-rank test was used to evaluate the effect of clinicopathological features on survival. Hazard ratio and $95 \%$ confidence interval were calculated using multivariate COX regression analyses. All statistical tests were two-sided, and $\mathrm{P}<0.05$ was considered to be statistically significant. The SPSS software (version 19.0) was utilized for statistical analysis.

Table 1. Difference in LMR according to clinical characteristics in ESCC patients

\begin{tabular}{|c|c|c|c|}
\hline & & \multicolumn{2}{|l|}{ Cases } \\
\hline \multicolumn{2}{|l|}{ Variables } & $\mathrm{N}$ & $\%$ \\
\hline \multirow[t]{2}{*}{ Sex } & Male & 582 & 85.6 \\
\hline & Female & 98 & 14.4 \\
\hline \multirow{2}{*}{$\begin{array}{l}\text { Age at therapy initiation } \\
\text { (years) }\end{array}$} & Median & 61 & \\
\hline & Interquartile range & $(56-67)$ & \\
\hline \multirow[t]{5}{*}{ Pathology grade } & Well differentiated & 49 & 7.2 \\
\hline & middle differentiated & 449 & 66.0 \\
\hline & Poorly differentiated & 166 & 24.4 \\
\hline & Undifferentiated & 2 & 0.3 \\
\hline & Missing & 14 & 2.1 \\
\hline \multirow[t]{3}{*}{ Depth of tumor } & T1a-1b & 64 & 9.4 \\
\hline & $\mathrm{T} 2$ & 131 & 19.3 \\
\hline & $\mathrm{T} 3$ & 485 & 71.3 \\
\hline \multirow[t]{4}{*}{ Lymph node metastasis } & N0 & 296 & 43.5 \\
\hline & N1 & 213 & 31.3 \\
\hline & N2 & 118 & 17.4 \\
\hline & N3 & 53 & 7.8 \\
\hline \multirow[t]{3}{*}{ Pathological stage } & $1 a-1 b$ & 116 & 17.1 \\
\hline & $2 a-2 b$ & 229 & 33.7 \\
\hline & $3 a-3 c$ & 335 & 49.3 \\
\hline \multirow[t]{2}{*}{ Vessel invasive } & Yes & 210 & 30.9 \\
\hline & No & 470 & 69.1 \\
\hline \multirow[t]{2}{*}{ Nerve infiltration } & Yes & 254 & 37.4 \\
\hline & No & 426 & 62.6 \\
\hline \multirow[t]{3}{*}{ Treatment regimen } & S & 461 & 67.8 \\
\hline & $S$ plus postoperative $C$ & 155 & 22.8 \\
\hline & $\mathrm{S}$ plus postoperative $\mathrm{CRT}$ & 64 & 9.4 \\
\hline \multirow{2}{*}{$\begin{array}{l}\text { Hospital time after operation } \\
\text { (days) }\end{array}$} & Median & 11 & \\
\hline & Interquartile range & \multicolumn{2}{|c|}{$(10-14)$} \\
\hline \multirow[t]{2}{*}{ LMR } & Median & \multicolumn{2}{|c|}{3.17} \\
\hline & Interquartile range & \multicolumn{2}{|c|}{$(2.33-4.33)$} \\
\hline
\end{tabular}

Abbreviations: S: surgery; C: chemotherapy; CRT: chemoradiotherapy; LMR: lymphocyte to monocyte ratio.

\section{Results}

\section{Patient characteristics}

We enrolled 680 patients with ESCC in this study, including $582(85.6 \%)$ males and 98 (14.4\%) females. The median age at therapy initiation was 61 years (Interquartile range: 56-67 years). There were $461(67.8 \%)$ patients received surgery without any other auxiliary treatment, $155(22.8 \%)$ patients received chemotherapy and $64(9.4 \%)$ had access to 
radiotherapy after the operation. The clinical characteristics were shown in Table 1.

Table 2. Relationship between preoperative LMR and clinicopathological features in patients with ESCC

\begin{tabular}{|c|c|c|c|c|}
\hline Characteristics & Total patients & $\begin{array}{l}\mathrm{LMR}<3.17 \\
(\mathrm{n}=343)\end{array}$ & $\begin{array}{l}\mathrm{LMR} \geq 3.17 \\
(\mathrm{n}=337)\end{array}$ & $P$ value \\
\hline \multirow[t]{2}{*}{ Sex } & Male & 321 & 261 & $<0.001$ \\
\hline & Female & 23 & 75 & \\
\hline \multirow{2}{*}{$\begin{array}{l}\text { Age at therapy } \\
\text { initiation(years) }\end{array}$} & $\leq 60$ & 148 & 164 & 0.130 \\
\hline & $>60$ & 196 & 172 & \\
\hline \multirow[t]{5}{*}{ Pathology grade } & Well differentiated & 28 & 21 & \\
\hline & middle differentiated & 228 & 221 & 0.539 \\
\hline & Poorly differentiated & 81 & 85 & \\
\hline & Undifferentiated & 0 & 2 & \\
\hline & Missing & 7 & 7 & \\
\hline \multirow[t]{3}{*}{ Depth of tumor } & $\mathrm{T} 1 \mathrm{a}-1 \mathrm{~b}$ & 25 & 39 & \\
\hline & $\mathrm{T} 2$ & 67 & 64 & 0.151 \\
\hline & T3 & 252 & 233 & \\
\hline \multirow{4}{*}{$\begin{array}{l}\text { Lymph node } \\
\text { metastasis }\end{array}$} & No & 148 & 148 & \\
\hline & N1 & 102 & 111 & 0.307 \\
\hline & N2 & 61 & 57 & \\
\hline & N3 & 33 & 20 & \\
\hline \multirow{3}{*}{$\begin{array}{l}\text { Pathological } \\
\text { stage }\end{array}$} & $1 a-1 b$ & 55 & 61 & \\
\hline & $2 a-2 b$ & 113 & 116 & 0.572 \\
\hline & $3 a-3 c$ & 176 & 159 & \\
\hline \multirow[t]{2}{*}{ Vessel invasive } & Yes & 106 & 104 & 0.969 \\
\hline & No & 238 & 232 & \\
\hline \multirow[t]{2}{*}{ Nerve infiltration } & Yes & 127 & 127 & 0.813 \\
\hline & No & 217 & 209 & \\
\hline \multirow{3}{*}{$\begin{array}{l}\text { Treatment } \\
\text { regimen }\end{array}$} & S & 234 & 227 & \\
\hline & $\mathrm{S}$ plus postoperative $\mathrm{C}$ & 81 & 74 & 0.641 \\
\hline & $\begin{array}{l}\text { S plus postoperative } \\
\text { CRT }\end{array}$ & 29 & 35 & \\
\hline \multirow{2}{*}{$\begin{array}{l}\text { Hospital time } \\
\text { after } \\
\text { operation(days) }\end{array}$} & $\leq 14$ & 264 & 273 & 0.149 \\
\hline & $>14$ & 80 & 63 & \\
\hline
\end{tabular}

Abbreviations: S: surgery; C: chemotherapy; CRT: chemoradiotherapy; LMR: lymphocyte to monocyte ratio.

The median of the LMR was 3.17(Interquartile range: $2.33-4.33$ ). We chose the median as the cutoff value and divided the patients into low LMR group $(\mathrm{LMR} \ll 3.17)$ and high LMR group (LMR $\geq 3.17)$. Thus, 343 patients with the low LMR and 337 patients were grouped into the high LMR.

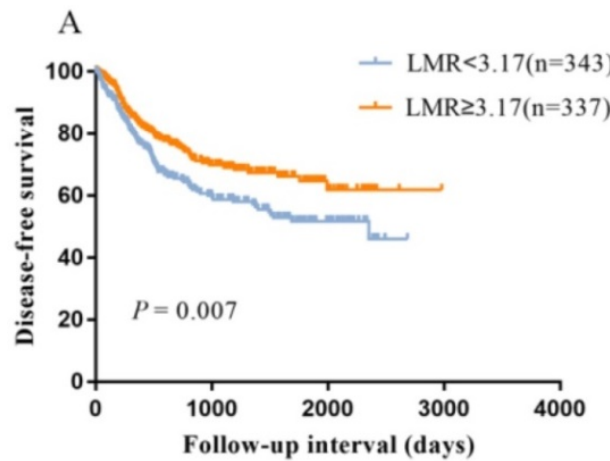

\section{Correlation between LMR and clinical features}

The correlation between LMR and clinical characteristics is listed in Table 2. None of the clinical features was significantly associated with the LMR including age at therapy initiation, pathology grade, depth of tumor, lymph node metastasis, pathological stage, vessel invasive, nerve infiltration, treatment regimen and hospital time after operation. Nevertheless, a decreased LMR was notably correlated with sex $(\mathrm{P}<0.001)$. The LMR was negatively correlated with sex $(\mathrm{r}=-0.245, \mathrm{P}<0.001)$ using Spearman analysis (Table 3 ).

Table 3. Spearman analysis of correlation between preoperative LMR and clinicopathological features

\begin{tabular}{lll}
\hline Variables & LMR & \\
\cline { 2 - 3 } & spearman correlation & $P$ value \\
\hline Sex & -0.245 & $<0.001$ \\
Age at therapy initiation(years) & -0.061 & 0.110 \\
Depth of tumor & -0.054 & 0.161 \\
Lymph node metastasis & -0.026 & 0.506 \\
Pathological stage & -0.037 & 0.335 \\
Vessel invasive & 0.008 & 0.825 \\
Nerve infiltration & 0.004 & 0.927 \\
Treatment regimen & 0.001 & 0.977 \\
Hospital time after operation(days) & 0.013 & 0.742
\end{tabular}

\section{Prognostic variables for DFS and OS}

As shown in Figure 1, the Kaplan-Meier curves indicated that patients with low LMR had a worse DFS ( $\mathrm{P}=0.007$, Figure 1A) and OS $(\mathrm{P}=0.018$, Figure $1 \mathrm{~B})$ compared with high LMR group.

Univariate analysis revealed that in addition to LMR $(\mathrm{P}=0.020)$, lymph node metastasis $(\mathrm{P}<0.001)$, pathological stage $(\mathrm{P}<0.001)$, vessel invasive $(\mathrm{P}=0.027)$, nerve infiltration $(\mathrm{P}<0.001)$ and treatment regimen $(\mathrm{P}<0.001)$ were also significantly correlated with DFS. After adjustment for confounders, lymph node metastasis $(\mathrm{P}<0.001)$, nerve infiltration $(\mathrm{P}=0.003)$, treatment regimen $(\mathrm{P}<0.001)$ and $\mathrm{LMR}(\mathrm{P}=0.003)$ were significantly associated with DFS (Table 4).

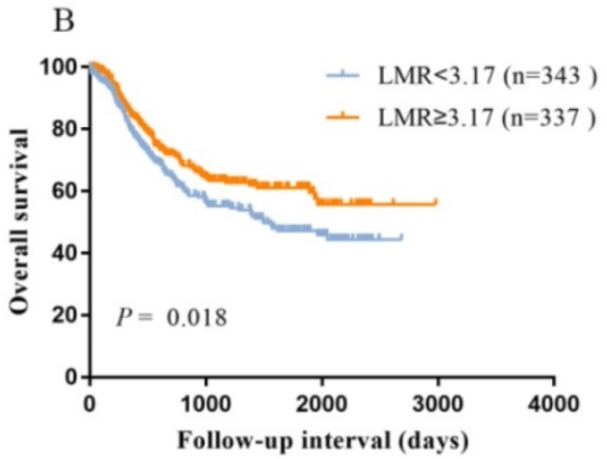

Figure 1. Kaplan-Meier survival curves showing the relationship between lymphocyte to monocyte ratio (LMR) and ESCC. A: Disease-free survival according to LMR; B: Overall survival according to LMR. 
Table 4. Disease-free survival analyses according to preoperative LMR in 680 patients with ESCC.

\begin{tabular}{|c|c|c|c|c|c|c|}
\hline \multirow[t]{2}{*}{ Variables } & \multicolumn{4}{|c|}{ Univariate } & \multicolumn{2}{|l|}{ Multivariate } \\
\hline & HR & $95 \% \mathrm{CI}$ & $P$ value & $\mathrm{HR}$ & $95 \%$ CI & $P$ value \\
\hline Sex (male vs. female) & 1.246 & $0.826-1.879$ & 0.295 & & & \\
\hline Age $(>60$ vs. $\leq 60)$ & 0.995 & $0.977-1.012$ & 0.559 & & & \\
\hline Depth of tumor (3 vs. 1+2) & 1.143 & $0.909-1.439$ & 0.253 & & & \\
\hline Lymph node metastasis (+ vs. -) & 1.667 & $1.453-1.910$ & $<0.001$ & 1.648 & $1.314-2.068$ & $<0.001$ \\
\hline Pathological stage (3 vs. $1+2$ ) & 1.64 & $1.332-2.021$ & $<0.001$ & 0.84 & $0.611-1.157$ & 0.286 \\
\hline Vessel invasive (+ vs. -) & 1.382 & $1.037-1.842$ & 0.027 & 0.81 & $0.578-1.136$ & 0.222 \\
\hline Nerve infiltration (+ vs. -) & 1.643 & $1.250-2.159$ & $<0.001$ & 1.57 & $1.160-2.126$ & 0.003 \\
\hline $\begin{array}{l}\text { Treatment regimen } \\
\text { (S vs. S+CRT/C) }\end{array}$ & 1.306 & $1.170-1.458$ & $<0.001$ & 1.299 & $1.147-1.471$ & $<0.001$ \\
\hline Hospital time after operation(days) (>14 vs. $\leq 14)$ & 1.003 & $0.989-1.017$ & 0.716 & & & \\
\hline LMR $(\geq 3.17$ vs. $<3.17)$ & 0.892 & $0.810-0.982$ & 0.020 & 0.854 & $0.768-0.949$ & 0.003 \\
\hline
\end{tabular}

Abbreviations: S: surgery; C: chemotherapy; CRT: chemoradiotherapy; LMR: lymphocyte to monocyte ratio.

Table 5. Overall survival analyses according to preoperative LMR in 680 patients with ESCC.

\begin{tabular}{|c|c|c|c|c|c|c|}
\hline \multirow[t]{2}{*}{ Variables } & \multicolumn{4}{|c|}{ Univariate } & \multicolumn{2}{|l|}{ Multivariate } \\
\hline & HR & $95 \% \mathrm{CI}$ & $P$ value & HR & $95 \%$ CI & $P$ value \\
\hline Sex (male vs. female) & 1.242 & $0.836-1.844$ & 0.283 & & & \\
\hline Age $(>60$ vs. $\leq 60)$ & 1.001 & 0.984-1.018 & 0.926 & & & \\
\hline Depth of tumor (3 vs. $1+2$ ) & 1.513 & $1.178-1.943$ & 0.001 & 0.987 & $0.714-1.364$ & 0.935 \\
\hline Lymph node metastasis (+ vs. -) & 1.755 & $1.544-1.995$ & $<0.001$ & 1.692 & $1.317-2.173$ & $<0.001$ \\
\hline Pathological stage (3 vs. $1+2$ ) & 1.929 & $1.563-2.382$ & $<0.001$ & 0.909 & $0.605-1.365$ & 0.644 \\
\hline Vessel invasive (+ vs. -) & 1.783 & $1.369-2.324$ & $<0.001$ & 0.885 & $0.634-1.237$ & 0.475 \\
\hline Nerve infiltration (+ vs. -) & 1.829 & $1.411-2.371$ & $<0.001$ & 1.524 & $1.12-2.075$ & 0.007 \\
\hline Treatment regimen (S vs. S+CRT/C) & 1.006 & $0.896-1.13$ & 0.916 & & & \\
\hline Hospital time after operation(days) (>14 vs. $\leq 14$ ) & 1.007 & 0.995-1.019 & 0.234 & & & \\
\hline $\operatorname{LMR}(\geq 3.17$ vs. $<3.17)$ & 0.895 & $0.816-0.981$ & 0.018 & 0.864 & $0.779-0.958$ & 0.006 \\
\hline
\end{tabular}

Abbreviations: S: surgery; C: chemotherapy; CRT: chemoradiotherapy; LMR: lymphocyte to monocyte ratio.

In the univariate analysis, we found that LMR was significantly associated with OS $(\mathrm{P}=0.018)$. Meanwhile, there was a close relationship between depth of tumor $(\mathrm{P}=0.001)$, lymph node metastasis $(\mathrm{P}<0.001)$, pathological stage $(\mathrm{P}<0.001)$, vessel invasive $(\mathrm{P}=0.027)$, nerve infiltration $(\mathrm{P}<0.001)$ and monocyte $(\mathrm{P}=0.022)$. In the multivariate analysis, lymph node metastasis $(\mathrm{P}<0.001)$, nerve infiltration $(\mathrm{P}=0.007)$ and LMR $(\mathrm{P}=0.006)$ were significantly related to OS (Table 5). Above all, LMR was an independent prognostic factor for ESCC after esophageal radical surgery.

\section{Discussions}

Up to now the association between preoperative LMR and the prognosis of patients with ESCC remains inconclusive. Therefore, we conducted this retrospective study concerning the patients without preoperative adjuvant treatment, which have an impact on the count and percentage of lymphocyte and monocyte. In the present research, low LMR was discovered to be significantly related to sex $(\mathrm{P}<0.001)$, with $70 \%$ of patients being male. There is a 2 -fold to 3 -fold difference in incidence and mortality rates between the gender worldwide [19]. A total of 731 patients newly diagnosed with ESCC from Feb 2008 to Jan 2015 at the Zhejiang Cancer Hospital were selected for the present study. With 51 patients excluded, 680 patients undergoing radical surgery were enrolled. Therefore, there was a possibility that decreased LMR was significantly associated with gender. The reason for no relationship between clinical features and LMR was that the patients undergoing radical surgery were in early and middle stage but not in advanced stage. Low LMR was correlated with the unfavorable prognosis of ESCC after radical surgery. We concluded that LMR was an independent prognostic factor for ESCC by using the Cox regression model.

The system inflammation in the tumor microenvironment has an impact on the tumorigenesis and progression. There were increasing studies suggest that the inflammation-based score were associated with the outcome of various cancers [20-25]. The representative markers of system inflammation are the peripheral inflammatory cells such as neutrophils, lymphocytes and monocytes. LMR ratio is the calculated value between lymphocytes and monocytes. As result, LMR ratio that is correlated with worse prognosis of patients with ESCC is on behalf of lymphocytes and monocytes. In other words, LMR presents tumorigenesis and progression through lymphocytes and monocytes. Lymphocytes play a major role in suppressing cancer cell proliferation and migration [26]. Besides, cytotoxic lymphocytes, mainly cytotoxic $\mathrm{T}$ cells, are essential for eliminating residual cancer 
cell and being applied in immunotherapy [27-28].

Monocytes are thought to have an impact on tumorigenesis through differentiation to tumor-associated macrophages (TAMs). TAMs are recruited to the tumor site by obtaining the signal from tumor-derived chemotactic factors [29]. Therefore, the mount and percentage of monocytes could represent for TAMs reflecting the tumor burden. Recent studies reported that increased infiltration of TAMs was associated with the outcome of various cancers [30-31]. Above all, LMR may substitute for the balance between the promotion of tumor reaction and anti-tumor immune response.

There was also a study that focused on the relationship between LMR and outcome in ESCC patients after radical surgery. The author concluded that low LMR $(\mathrm{LMR} \leq 4.0)$ was correlated with the worse cancer specific survival and overall survival. The clinical information was based on 147 cases of ESCC patients in Japan. Besides, the cut off value was determined by the Receiver operating characteristics (ROC) curve analysis [16]. In our study, we chose the median value of LMR as the optimal cut off value. Disease free survival and overall survival represented the survival of ESCC patients. Based on 680 cases patients with primary diagnosis of ESCC, we found that low LMR was an independent prognostic factor in ESCC patients in China.

There were several limitations of this study: first, it was the single-center design and retrospective analysis. Second, it was uncertain whether a different cut off value determined by other ways including ROC curve analysis and $C$ index would serve as an optimal indicator of survival in ESCC patients. The cut off value of LMR ranged from 2.93 to 5.3 in the reported studies. We chose the median value of LMR, which minimized a false positive result, as the cut off value. Therefore, multi-center design and prospective trials are required to confirm the reproducibility of these findings.

In summary, a low preoperative LMR indicates a worse survival of patients with primary diagnosis ESCC who received radical surgery. This finding may help clinicians to assess the prognosis of ESCC patients after operation.

\section{Acknowledgements}

We thank the included patients and all the investigators, including the clinicians and laboratory technicians in our study. This study was funded by National Natural Science Foundation of China (contract/grant number: 81502603), General research program of Health Department of Zhejiang Province (contract/grant number: 2016KYB048) and Zhejiang
Youth Talents Project (contract/grant number: 2019RC026).

\section{Competing Interests}

The authors have declared that no competing interest exists.

\section{References}

1. McGuire S. World Cancer Report 2014. Geneva, Switzerland: World Health Organization, International Agency for Research on Cancer, WHO Press, 2015. Advances in nutrition. 2016; 7: 418-9.

2. Kamangar F, Dores GM, Anderson WF. Patterns of cancer incidence, mortality, and prevalence across five continents: defining priorities to reduce cancer disparities in different geographic regions of the world. Journal of clinical oncology. 2006; 24: 2137-50.

3. Tran GD, Sun XD, Abnet CC, et al. Prospective study of risk factors for esophageal and gastric cancers in the Linxian general population trial cohort in China. International journal of cancer. 2005; 113: 456-63.

4. Gertler R, Stein HJ, Langer R, et al. Long-term outcome of 2920 patients with cancers of the esophagus and esophagogastric junction: evaluation of the New Union Internationale Contre le Cancer/American Joint Cancer Committee staging system. Annals of surgery. 2011; 253: 689-98.

5. Jemal A, Siegel R, Ward E, et al. Cancer statistics, 2007. CA. 2007; 57: 43-66.

6. Allum WH, Stenning SP, Bancewicz J, et al. Long-term results of a randomized trial of surgery with or without preoperative chemotherapy in esophageal cancer. Journal of clinical oncology. 2009; 27: 5062-7.

7. Washington K. 7th edition of the AJCC cancer staging manual: stomach. Annals of surgical oncology. 2010; 17: 3077-9.

8. Grivennikov SI, Greten FR, Karin M. Immunity, inflammation, and cancer. Cell. 2010; 140: 883-99.

9. Minami S, Ihara S, Kim SH, et al. Lymphocyte to Monocyte Ratio and Modified Glasgow Prognostic Score Predict Prognosis of Lung Adenocarcinoma Without Driver Mutation. World journal of oncology. 2018; 9: 13-20.

10. Saito H, Kono $\mathrm{Y}$, Murakami $\mathrm{Y}$, et al. Prognostic Significance of the Preoperative Ratio of C-Reactive Protein to Albumin and Neutrophil-Lymphocyte Ratio in Gastric Cancer Patients. World journal of surgery. 2018; 42: 1819-25.

11. Ma J, Kuzman J, Ray A, et al. Neutrophil-to-lymphocyte Ratio (NLR) as a predictor for recurrence in patients with stage III melanoma. Scientific reports. 2018; 8: 4044.

12. Yang T, Zhu J, Zhao L, et al. Lymphocyte to monocyte ratio and neutrophil to lymphocyte ratio are superior inflammation-based predictors of recurrence in patients with hepatocellular carcinoma after hepatic resection. Journal of surgical oncology. 2017; 115: 718-28.

13. Marin Hernandez C, Pinero Madrona A, Gil Vazquez PJ, et al. Usefulness of lymphocyte-to-monocyte, neutrophil-to-monocyte and neutrophil-to-lymphocyte ratios as prognostic markers in breast cancer patients treated with neoadjuvant chemotherapy. Clinical \& translational oncology. 2018; 20: 476-83.

14. Zhu JY, Liu CC, Wang L, et al. Peripheral blood lymphocyte-to-monocyte ratio as a prognostic factor in advanced epithelial ovarian cancer: a multicenter retrospective study. Journal of Cancer. 2017; 8: 737-43.

15. Huang Y, Feng JF. Low preoperative lymphocyte to monocyte ratio predicts poor cancer-specific survival in patients with esophageal squamous cell carcinoma. OncoTargets and therapy. 2015; 8: 137-45.

16. Hirahara N, Matsubara T, Kawahara D, et al. Prognostic significance of preoperative inflammatory response biomarkers in patients undergoing curative thoracoscopic esophagectomy for esophageal squamous cell carcinoma. European journal of surgical oncology. 2017; 43: 493-501.

17. Wang L, Wang C, Wang J, et al. A novel systemic immune-inflammation index predicts survival and quality of life of patients after curative resection for esophageal squamous cell carcinoma. Journal of cancer research and clinical oncology. 2017; 143: 2077-86.

18. Zhu $\mathrm{Y}, \mathrm{Li} \mathrm{M}$, Bo $\mathrm{C}$, et al. Prognostic significance of the lymphocyte-to-monocyte ratio and the tumor-infiltrating lymphocyte to tumor-associated macrophage ratio in patients with stage T3N0M0 esophageal squamous cell carcinoma. Cancer immunology, immunotherapy : CII. 2017; 66: 343-54

19. Bray F, Ferlay J, Soerjomataram I, et al. Global cancer statistics 2018: GLOBOCAN estimates of incidence and mortality worldwide for 36 cancers in 185 countries. CA. 2018.

20. Zhang H, Lu J, Lu Y, et al. Prognostic significance and predictors of the system inflammation score in ovarian clear cell carcinoma. PloS one. 2017; 12: e0177520.

21. Tan D, Fu Y, Tong W, et al. Prognostic significance of lymphocyte to monocyte ratio in colorectal cancer: A meta-analysis. International journal of surgery. 2018; 55: 128-38 
22. Tham T, Olson C, Khaymovich J, et al. The lymphocyte-to-monocyte ratio as a prognostic indicator in head and neck cancer: a systematic review and meta-analysis. European archives of oto-rhino-laryngology. 2018; 275: 1663-70.

23. Ma JY, Liu Q. Clinicopathological and prognostic significance of lymphocyte to monocyte ratio in patients with gastric cancer: A meta-analysis. International journal of surgery. 2018; 50: 67-71.

24. Hu RJ, Ma JY, Hu G. Lymphocyte-to-monocyte ratio in pancreatic cancer: Prognostic significance and meta-analysis. Clinica chimica acta. 2018; 481: 142-6.

25. Hu RJ, Liu Q, Ma JY, et al. Preoperative lymphocyte-to-monocyte ratio predicts breast cancer outcome: A meta-analysis. Clinica chimica acta. 2018; 484: 1-6.

26. Bastid J, Bonnefoy N, Eliaou JF, et al. Lymphocyte-derived interleukin-17A adds another brick in the wall of inflammation-induced breast carcinogenesis. Oncoimmunology. 2014; 3: e28273.

27. Nazir T, Islam A, Omer MO, et al. Lymphocytopenia; induced by vinorelbine, doxorubicin and cisplatin in human cancer patients. Breast disease. 2015; 35: $1-4$.

28. Martinez-Lostao L, Anel A, Pardo J. How Do Cytotoxic Lymphocytes Kill Cancer Cells? Clinical cancer research. 2015; 21: 5047-56.

29. Chanmee T, Ontong P, Konno K, et al. Tumor-associated macrophages as major players in the tumor microenvironment. Cancers. 2014; 6: 1670-90.

30. Yang M, Li Z, Ren M, et al. Stromal Infiltration of Tumor-Associated Macrophages Conferring Poor Prognosis of Patients with Basal-Like Breast Carcinoma. Journal of Cancer. 2018; 9: 2308-16.

31. Zhang WJ, Wang XH, Gao ST, et al. Tumor-associated macrophages correlate with phenomenon of epithelial-mesenchymal transition and contribute to poor prognosis in triple-negative breast cancer patients. The Journal of surgical research. 2018; 222: 93-101. 